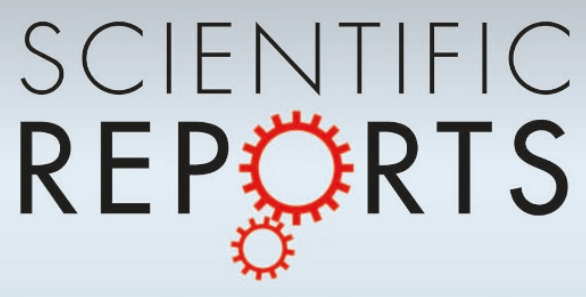

\title{
Heteroepitaxial Growth of Single-Walled Carbon Nanotubes from Boron Nitride
}

SUBJECT AREAS:

CARBON NANOTUBES

AND FULLERENES

SYNTHESIS AND PROCESSING

TRANSMISSION ELECTRON

MICROSCOPY

ATOMISTIC MODELS

Received

10 August 2012

Accepted

26 November 2012

Published

13 December 2012

Correspondence and requests for materials should be addressed to

C.L. (cliu@imr.ac.cn)

* These authors contributed equally to this work.

\author{
Dai-Ming Tang 1,3*, Li-Li Zhang ${ }^{1 *}$, Chang Liu' , Li-Chang Yin' ', Peng-Xiang Hou' ' Hua Jiang ${ }^{2}$, Zhen Zhu' \\ Feng Li', Bilu Liu', Esko I. Kauppinen² \& Hui-Ming Cheng'
}

'Shenyang National Laboratory for Materials Science, Institute of Metal Research, Chinese Academy of Sciences, 72 Wenhua Road, Shenyang 1 10016, China, ${ }^{2}$ NanoMaterials Group, Department of Applied Physics and Center for New Materials, Aalto University, Puumiehenkuja 2, 00076, Finland, ${ }^{3}$ Present addresses: International Center for Materials Nanoarchitectonics (MANA), National Institute for Materials Science (NIMS), Namiki 1-1, Tsukuba, lbaraki, 305-0044 Japan.

The growth of single-walled carbon nanotubes (SWCNTs) with predefined structure is of great importance for both fundamental research and their practical applications. Traditionally, SWCNTs are grown from a metal catalyst with a vapor-liquid-solid mechanism, where the catalyst is in liquid state with fluctuating structures, and it is intrinsically unfavorable for the structure control of SWCNTs. Here we report the heteroepitaxial growth of SWCNTs from a platelet boron nitride nanofiber (BNNF), which is composed of stacked (002) planes and is stable at high temperatures. SWCNTs are found to grow epitaxially from the open (002) edges of the BNNFs, and the diameters of the SWCNTs are multiples of the BN (002) interplanar distance. In situ transmission electron microscopy observations coupled with first principles calculations reveal that the growth of SWCNTs from the BNNFs follows a vapor-solid-solid mechanism. Our work opens opportunities for the control over the structure of SWCNTs by hetero-crystallographic epitaxy.

recise control of the structure of single-walled carbon nanotubes (SWCNTs) is crucial for their applications in high-performance nanodevices, since the electrical properties of SWCNTs are strongly dependent on their diameter and chiral angle ${ }^{1,2}$. In general, SWCNTs are grown from transitional metal catalyst nanoparticles (NPs) using a vapor-liquid-solid (VLS) growth mechanism. It is recognized that the structure of a SWCNT is largely determined by the size, morphology, and composition of the catalyst from which it nucleates and grows ${ }^{3-5}$. Great efforts have been made to control the structure of SWCNTs by catalyst engineering, and notable progress has been made on positioning ${ }^{6}$, alignment ${ }^{7}$, length ${ }^{8}$ and diameter ${ }^{9}$ control and the preferential production of specific conductivities ${ }^{10-14}$ and chiralities ${ }^{15-18}$. However, the synthesis of SWCNTs with a predefined and precisely controlled structure still remains a big challenge. One important reason is that during the VLS growth process, metal catalyst particles are in a liquid or partial liquid state with fluctuating sizes, morphologies and crystal structures ${ }^{19-23}$, which makes it difficult to obtain SWCNTs with the desired uniformity and controllability.

Recently, it was found that some non-metal materials can be used as catalysts to grow SWCNTs. A distinct feature of the non-metal catalysts is that their structure and morphology remain unchanged during the growth process even at high temperatures, due to their much higher melting points compared with traditional metal catalysts, and this may facilitate controlled growth of SWCNTs. For example, it was found that the SWCNTs grown from nanodiamonds have polyhedron-shaped caps rather than the usual hemispherical ones ${ }^{19}$. SWCNTs with discretely distributed diameters were produced by using opened fullerenes as the catalyst ${ }^{20}$. A "cloning" growth of SWCNTs from short, end-opened SWCNTs was also reported, and the chirality of the newly grown parts is exactly the same as that of the original SWCNT fragment ${ }^{21}$. The above work shows that the structure of SWCNTs could be closely correlated with the non-metal catalysts used.

Here, we develop a heteroepitaxial growth approach for preparing SWCNTs with predefined structures from a platelet boron nitride nanofiber (BNNF) as the growth seed. The BNNF has a graphite-like structure with (002) planes stacked perpendicular to the fiber axis. SWCNTs are found to grow epitaxially from the open BN (002) edges, and their diameters are multiples of the BN (002) interplanar distance. The growth process and mechanism were investigated by both in situ TEM observations and first principles calculations. Our results indicate that structure control of SWCNTs can be realized by using a catalyst with good structural stability and specific crystal structure by epitaxial growth. 


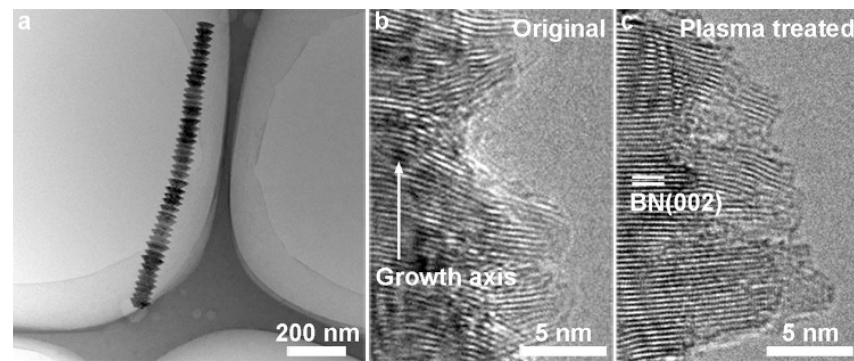

Figure $1 \mid$ TEM images of the (a, b) original and (c) oxygen-plasma treated BNNFs. $(\mathrm{a}, \mathrm{b})$ The BNNFs are composed of stacked platelets, and the BN (002) planes are oriented perpendicular to the fiber axis, with neighboring $\mathrm{BN}$ layers connecting with each other by forming small protrusions. (c) These closed protrusions are preferentially etched by the oxygen-plasma treatment to open (002) edges.

\section{Results}

Growth of SWCNTs from BNNFs. The BNNFs were prepared using a floating catalyst CVD method ${ }^{22,23}$. As shown in Fig. 1a, the BNNFs have stacked-platelet morphology and a mean diameter of around $130 \mathrm{~nm}$. EDS (Fig. S1) analysis shows that they are composed of only boron and nitrogen. A HRTEM image of a BNNF is shown in Fig. 1b. It can be seen that the (002) planes of $\mathrm{BN}$ are oriented perpendicular to the growth axis. As a result of this special stacking, the bonds at the surface of the BNNFs are unsaturated. To reduce surface energy, neighboring $\mathrm{BN}$ layers tend to connect with each other to form protrusions. In our experiments, the original BNNFs were found to be inefficient for growing SWCNTs and an oxygen plasma treatment was carried out to activate them. A TEM image of the BNNFs after plasma treatment is shown in Fig. 1c, and we can see that the closed edges have been etched, resulting in a high content of open $\mathrm{BN}(002)$ edges.

The obtained BNNFs were dispersed in ethanol and dropped onto a silicon substrate (with a natural $\mathrm{SiO}_{2}$ layer), and then put into a tubular furnace for SWCNT growth by the CVD at $900^{\circ} \mathrm{C}$. The morphology of the samples obtained was observed by SEM (Figs. 2a-b). It can be seen that the BNNF growth seeds retain their original morphology and no sintering of the BNNFs occurred, indicating that they have good stability at $900^{\circ} \mathrm{C}$. Some fibrous substance connecting with the BNNFs is observed in Fig. 2a. A higher magnification SEM image (Fig. 2b) shows that this fibrous material is rooted in the sharp protrusions of the BNNFs. A typical laser Raman spectrum of the products is shown in Fig. 2c. The characteristic Gmode peak at around $1590 \mathrm{~cm}^{-1}$ and the radial breathing mode (RBM) peaks in the range of $120-230 \mathrm{~cm}^{-1}$ indicate that the fibrous substances formed are SWCNTs. The peaks located at around $1320 \mathrm{~cm}^{-1}$ and $1360 \mathrm{~cm}^{-1}$ can be ascribed to the D mode of SWCNTs and the $\mathrm{G}$ mode of hexagonal $\mathrm{BN}$, respectively. It was found that the plasma treatment significantly improves the growth efficiency of SWCNTs (Fig. S2). Fig. S2 shows SEM images of SWCNTs obtained from the BNNFs with and without plasma treatment. In Fig. S2a, only sparse SWCNTs are observed, while under identical growth conditions, the area density of SWCNTs was increased several fold when the pre-treated BNNFs were used (Fig. S2b). By correlating the improved growth efficiency and the increased number of open edges caused by the oxygen plasma treatment (Fig. 1c), it is reasonable to assume that the open edges are active sites for growing SWCNTs. The products were further characterized using TEM (Figs. 2d-f). Figs. 2d and 2e show small SWCNT bundles (d) grown on the surface of a BNNF and (e) bridging two BNNFs. Along with the SWCNT bundle, a thin layer of deposited amorphous carbon is also observed (Fig. 2d). Special attention is paid to the root of the SWCNTs. As shown in Fig. 2f, a SWCNT is rooted in a BNNF, and the wall of the SWCNT is parallel to and connected to the $\mathrm{BN}$ (002) planes of the open edges, strongly indicating that the SWCNT is grown from the BNNF by an epitaxial mechanism.

$\mathrm{X}$-ray photoelectron spectroscopy (XPS) analysis was conducted for the SWCNTs grown from BNNFs, as shown in Fig. S3. Strong signals at $284.5 \mathrm{eV}$ from carbon are detected, and the peaks at $190.6 \mathrm{eV}$ and $397.8 \mathrm{eV}$ originate from boron and nitrogen, respectively. No metal impurities could be found. Electron energy loss
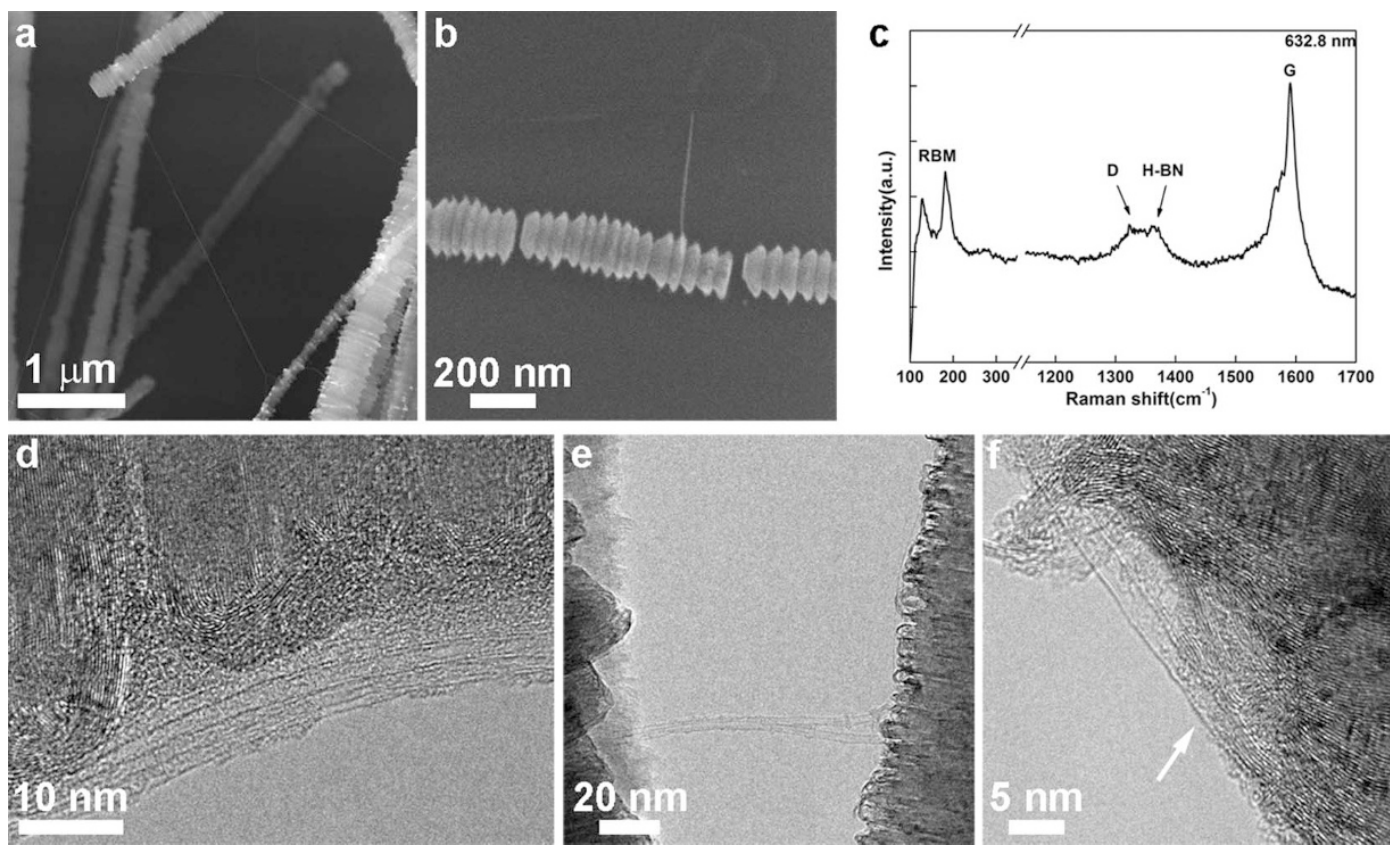

Figure 2 SEM, Raman and TEM characterizations of the SWCNTs grown from BNNFs. (a, b) SEM images of a sample after CVD growth. Both BNNFs and newly grown fibrous materials are observed. (c) Raman spectrum of the as-grown samples. RBM peaks that are characteristic of SWCNTs are clearly observed. (d-e) TEM images showing small SWCNT bundles grown along and bridging the BNNFs. (f) TEM image of a SWCNT rooted in a BNNF (indicated by the arrow), with its wall connected to the open BN (002) edges. 


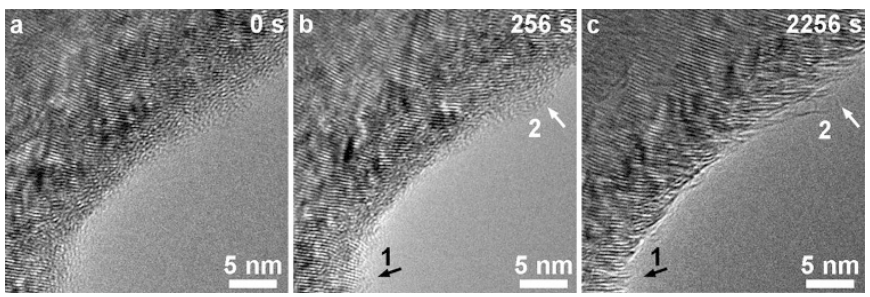

Figure 3 In situ TEM observations of the nucleation and growth of a SWCNT on the BNNF surface. (a) A thin layer of amorphous carbon is deposited on the BNNF surface. (b) Under Joule heating, the carbon layer tends to accumulate at position 2. (c) A short SWCNT with a diameter of $\sim 1.7 \mathrm{~nm}$ and a length of $\sim 8.2 \mathrm{~nm}$ grows at position 2 , with its wall connected to the open BN (002) edges.

spectroscopy (EELS) mapping was used to further reveal the elemental distribution (Fig. S4). Fig. S4a shows a BNNF with a length of $\sim 6 \mu \mathrm{m}$. In Fig. S4b, we can see a SWCNT grown from a protrusion of the BNNF. Fig. S4c and S4d show boron and nitrogen elemental distributions, and we can see that both elements are uniformly distributed with alternating bright and dark contrast along the fiber axis. Carbon is mainly located in the nanotube, and a very thin carbon layer can also be observed on the surface of the BNNF (Fig. S4e). The combined elemental map TEM images (Fig. S4f) clearly show that the SWCNT is rooted in the protrusion of the BNNF (as indicated by the arrow).

Heteroepitaxial growth mechanism. The above results show that SWCNTs can be grown from BNNFs, and the open (002) edges are presumed to be the active growth sites. To verify this assumption, in situ TEM observations and first principles calculations were carried out to investigate the growth mechanism.

We performed in situ growth of SWCNTs from BNNFs inside a TEM. First, a thin layer of amorphous carbon $(\sim 3 \mathrm{~nm}$ thick $)$ was deposited on the surface of the BNNFs by thermal decomposition of $\mathrm{C}_{2} \mathrm{H}_{2}$ at $700^{\circ} \mathrm{C}$ (Fig. 3a). Then the BNNFs were loaded in a TEMSTM holder and put into the TEM. One BNNF was selected and connected to a gold probe driven by a piezoelectric-tube. Because of the carbon layer, the BNNF becomes conductive. When a voltage is applied, a high temperature can be reached by Joule heating ${ }^{24-26}$. The structural evolution of the carbon layer on the BNNF surface was carefully monitored. When a DC current of $\sim 35 \mu \mathrm{A}$ was passed through the BNNF, the carbon distribution changes to be non-uniform (Fig. 3b) with the carbon layer becoming thinner at position 1, indicated by a black arrow, and accumulation of carbon is observed around position 2, marked by a white arrow. A more detailed observation shows that the $\mathrm{BN}$ layers at position 1 are closed, while open $\mathrm{BN}$ edges are observed at position 2 . The aggregated carbon gradually becomes ordered, and finally, a short SWCNT with a diameter $\sim 1.7 \mathrm{~nm}$ and a length $\sim 8.2 \mathrm{~nm}$ is observed to form (Fig. $3 \mathrm{c}$ ). It can be seen that the wall of the nanotube is connected to the open (002) edges of the BNNF. This in situ growth provides direct evidence that SWCNTs epitaxially grow from BNNFs. The open BN edges play an important role in the accumulation of carbon and the nucleation of SWCNTs.

First principles calculations were performed to investigate the driving force of the heteroepitaxial growth of SWCNTs from BNNFs. The structural model of the BNNFs was based on the HRTEM characterization. A $(5,5)$ SWCNT with a diameter of $0.68 \mathrm{~nm}$ was placed on a BN crystal with open (002) edges. Figs. $4 \mathrm{a}$ and $4 \mathrm{~b}$ show the structure models before and after relaxation. It can be seen that bonding is formed spontaneously between the SWCNT and the BN after relaxation. No obvious distortion is observed for both the SWCNT and BN. In the meantime, open (002) edges without SWCNTs attached are closed by forming bonding between adjacent unsaturated atoms. The
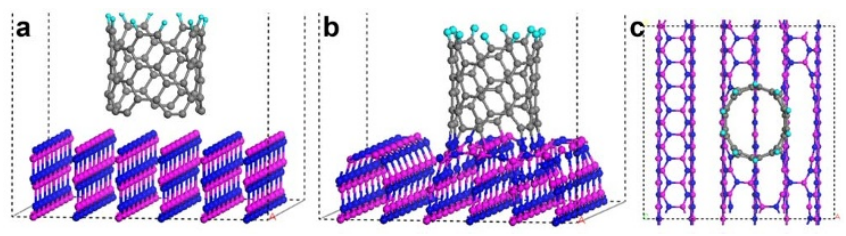

Figure 4 First principles calculations. Simulated structural model of a $(5,5)$ SWCNT grown on BN surface (a) before and (b, c) after structural optimization. The SWCNT spontaneously forms bonds with open (002) edges to reduce the total energy. Open edges without SWCNTs attached are closed by forming bonds between adjacent unsaturated atoms.

total energy is reduced by $89.523 \mathrm{eV}(8.952 \mathrm{eV} /$ bond $)$, indicating that the epitaxial growth of SWCNTs from BNNFs is energetically favored. The driving force can be ascribed to the reduction of dangling bonds by forming $\mathrm{B}-\mathrm{C}$ and $\mathrm{N}-\mathrm{C}$ bonds. Diffusion of carbon atoms on the $\mathrm{BN}$ crystal is another key step to ensure a sufficient carbon supply for the SWCNT nucleation and growth. Therefore, we further investigated the minimum energy path for carbon atom diffusion on a BNNF. The energy barriers obtained for the open and closed BN edges are $2.04 \mathrm{eV}$ and $1.29 \mathrm{eV}$, respectively, indicating that carbon atoms tend to diffuse over the closed edges and to be trapped by the open (002) edges. Our theoretical results fully support the heteroepitaxial growth mechanism, i.e. open (002) edges are active sites for the nucleation and growth of SWCNTs.

Base on the above experimental results and theoretical calculations, a growth mechanism of SWCNTs from BNNFs is proposed and illustrated in Fig. 5. (002) BN planes are perpendicular to the axis of a BNNF with both closed and open edges on its surface (Fig. 5a). At high temperatures, a thin layer of amorphous carbon is deposited on the surface by hydrocarbon decomposition (Fig. 5b). Carbon clusters form caps by forming bonds with the open $\mathrm{BN}$ edges to reduce the number of dangling bonds and the total energy of the system (Fig. 5c). Finally, SWCNTs grow from the nucleated caps with the carbon being supplied through adjacent surface diffusion (Fig. 5d). The diameter of the grown SWCNTs is determined by the number of layers of open (002) plane edges, from which the nanotube nucleates and grows.

Predefined diameter distribution of SWCNTs. The diameters of the SWCNTs obtained were characterized by both HRTEM and Raman spectroscopy (Fig. 6). As clearly shown in Fig. 6a, of the statistical analysis of more than 50 nanotubes, their diameters are mainly distributed in the range of $1 \mathrm{~nm}$ to $3 \mathrm{~nm}$. It is worth noting that the diameter distribution is discrete, showing obvious steps in multiples (4-7 times) of the (002) interplanar distance $(0.33 \mathrm{~nm})$ of $\mathrm{BN}$, which is consistent with the above proposed heteroepitaxial growth mechanism. Resonant Raman spectroscopy is a convenient method for the assessment of SWCNT diameters ${ }^{27}$. Fig. $6 \mathrm{~b}$ shows typical RBM bands of the as-grown SWCNTs, with an excitation wavelength of $632.8 \mathrm{~nm}$. It can be seen that the RBM bands are composed of a major peak at around $190 \mathrm{~cm}^{-1}$ corresponding to a diameter of $1.34 \mathrm{~nm}$, four times the $\mathrm{BN}$ (002) spacing, and a minor

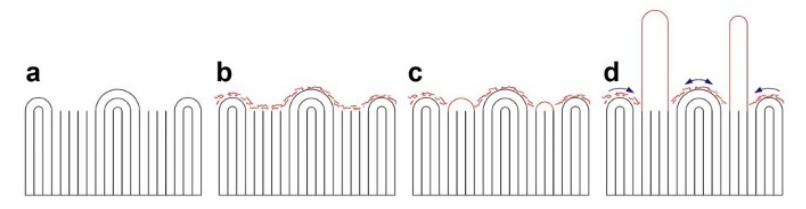

Figure $5 \mid$ Illustration of the heteroepitaxial growth process. (a) A pristine BNNF with both closed and open surface edges. (b) Amorphous carbon coating on the surface edges of the BNNF. (c) Formation of carbon caps at the open edges. (d) Epitaxial growth of SWCNTs. 

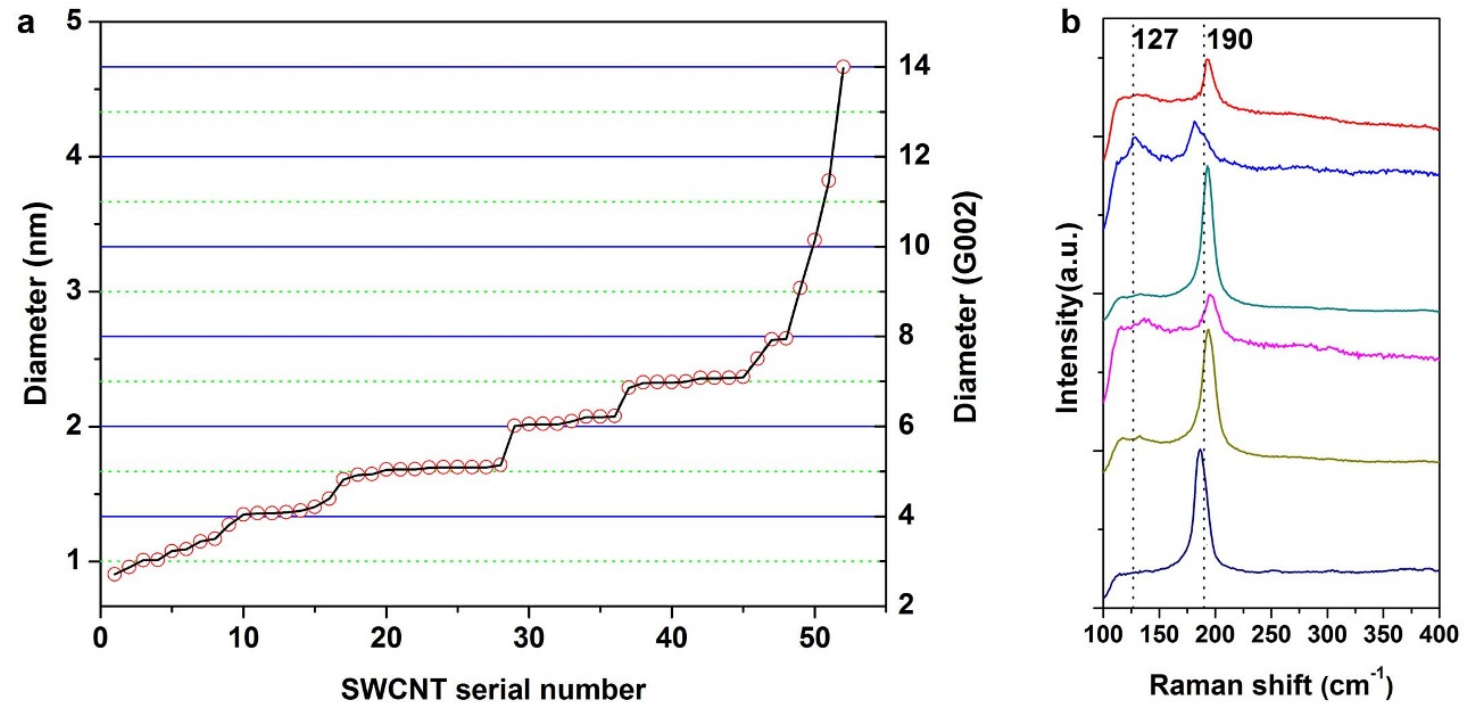

Figure 6 Diameter distribution of the SWCNTs grown from BNNFs. (a) Diameter distribution from HRTEM observations, demonstrating that the diameters of the SWCNTs are mostly distributed in discrete steps which correspond to multiples of the BN (002) interplanar spacing. (b) RBM peaks of the Raman spectra of SWCNTs (from six different samples), showing a major peak at around $190 \mathrm{~cm}^{-1}$ and a minor peak at around $127 \mathrm{~cm}{ }^{-1}$, corresponding to nanotube diameters of $1.34 \mathrm{~nm}$ and $2.00 \mathrm{~nm}$, respectively.

peak at around $127 \mathrm{~cm}^{-1}$, corresponding to a diameter of $2.00 \mathrm{~nm}$, six times the $\mathrm{BN}$ (002) spacing.

\section{Discussion}

Previous theoretical studies have emphasized the importance of the structure of catalysts and their interactions with carbon in the structure-controlled growth of SWCNTs ${ }^{3,5,28}$. It has been demonstrated that the chirality distribution of SWCNTs is strongly dependent on the composition of the catalyst, and the perturbations to the crystalline parameters such as interplanar distance ${ }^{29}$. In addition, chirality selective nucleation of carbon caps on metal surfaces has been proposed based on total energy calculations of lattice-matched or nonmatched models ${ }^{30}$. Obviously, the lattice-matched growth model, that is, epitaxy, requires a catalyst with a highly stable crystalline structure even at elevated growth temperatures. However, metal catalyst NPs are usually in a liquid or partial-liquid state when they catalytically grow SWCNTs by the VLS growth mechanism ${ }^{31-35}$. As a result, the SWCNTs grown from metal catalysts usually have variable and undefined diameters and structures. In contrast, non-metal catalysts have higher melting points and better structural stability ${ }^{26}$, and SWCNTs with discrete diameters have been produced from opened $\mathrm{C}_{60}$ fragments $^{20}$. The BNNF used here is thermally and chemically stable (Figs. 2-3), and more importantly, its predefined lamellar structure is effective for the epitaxial growth of SWCNTs. The diameters of the grown SWCNTs are discrete multiples of the BN (002) interplanar distance. Therefore, our work demonstrates an effective approach for the synthesis of SWCNTs with predefined diameters. This strategy can perhaps be further extended to layered materials with different interlayer distances, such as $\mathrm{BaTiO}_{3}(0.23 \mathrm{~nm}), \mathrm{Bi}_{2} \mathrm{Se}_{3}$ $(0.48 \mathrm{~nm})$, and $\mathrm{WS}_{2}(0.62 \mathrm{~nm})^{36-38}$. By using these layered compounds as growth seeds, SWCNTs with specific diameters or even chiralities can hopefully be obtained.

In summary, we have developed a heteroepitaxial growth approach for preparing SWCNTs using a BNNF as the growth seed. SWCNTs are observed to grow from protrusions on the BNNFs with their walls epitaxially extending from the open $\mathrm{BN}$ (002) edges. The diameters of the SWCNTs are multiples of the (002) interplanar distance of BN. First principles calculations reveal that the driving force of the SWCNT growth is the reduction of the number of dangling bonds and the total system energy. A growth process including carbon deposition, surface diffusion, cap formation and SWCNT growth at open (002) edges is proposed based on in situ TEM observations and theoretical calculations. This heteroepitaxial growth method opens new opportunities for the diameter and even chirality control of the growth of SWCNTs, by using lamellar materials with good structural stability and predefined crystal structure as growth seeds.

\section{Methods}

Growth and treatment of BNNFs. The BNNFs were produced using a floating catalyst CVD method, with amorphous boron and boron oxide powders as boron source, $\mathrm{NH}_{3}$ as nitrogen source, and ferrocene as catalyst precursor ${ }^{22,23}$. The asprepared BNNFs were soaked in concentrated hydrochloric acid at $80^{\circ} \mathrm{C}$ for $12 \mathrm{~h}$. After which the dispersion was centrifuged for $3 \mathrm{~min}(15000 \mathrm{r} / \mathrm{min})$. The remaining precipitate was then washed by de-ionized water and sonicated repeatedly. EDS and XPS characterizations were performed to make sure no trace of metals remains in the sample (Figs. S1 and S3). For plasma treatment, a BNNF sample dispersed in alcohol was dropped onto $\mathrm{a} \mathrm{SiO}_{2} / \mathrm{Si}$ wafer and then exposed to oxygen plasma for $20 \mathrm{~min}$ (Plasma Cleaner, PDC-32G).

CVD Growth of SWCNTs. A silicon substrate loaded with BNNFs was put into a quartz boat $(\Phi 30 \mathrm{~mm} \times 200 \mathrm{~mm})$ located at the center of a quartz reaction tube $(\Phi 45 \mathrm{~mm} \times 1000 \mathrm{~mm})$ and inserted into a horizontal tubular furnace. Thermal annealing was carried out at $700^{\circ} \mathrm{C}$ for $3 \sim 5 \mathrm{~min}$ in air, and the reaction chamber was evacuated to less than $30 \mathrm{~Pa}$, followed by the introduction of an Ar flow. The temperature was raised to $900^{\circ} \mathrm{C}$ under a mixed $\mathrm{Ar} / \mathrm{H}_{2}(200 \mathrm{sccm} / 400 \mathrm{sccm})$ gas flow for $15 \mathrm{~min}$. Ethanol vapor was then introduced by $\mathrm{Ar}(100 \mathrm{sccm})$ bubbling. The growth time was $15 \mathrm{~min}$. The reactor was cooled to room temperature under the $\mathrm{Ar} / \mathrm{H}_{2}(100 \mathrm{sccm} / 100 \mathrm{sccm})$ flow after growth.

Characterizations. The BNNFs and SWCNTs were characterized with scanning electron microscopy (SEM, Nova NanoSEM 430), high-resolution transmission electron microscopy (HRTEM, JEOL aberration-corrected 2200FS, FEI Tecnai F20) and Raman spectroscopy (Jobin Yvon HR800, with a $632.8 \mathrm{~nm}$ He-Ne laser).

In situ TEM observations of the nucleation and growth of SWCNTs from BNNFs. For carbon deposition, the purified BNNFs were placed in the center of a quartz tube reactor with a flow of $200 \mathrm{sccm}$ Ar. When the temperature was raised to $700^{\circ} \mathrm{C}, \mathrm{C}_{2} \mathrm{H}_{2}$ was introduced with a flow rate of $10 \mathrm{sccm}$. Deposition lasted for $30 \mathrm{~min}$ and the furnace was then cooled to room temperature under the protection of the Ar flow. The carbon-coated BNNFs were loaded on the end of a sharp gold wire, then the gold wire was assembled in a TEM-STM holder (Nanofactory Instruments AB) and put into a TEM (FEI Tecnai F20) chamber. A gold tip driven by a piezoelectric-tube was manipulated to connect with the selected BNNF. When a voltage was applied, a DC current passed through the carbon-coated BNNFs. Due to Joule heating, the temperature was increased to drive the growth of SWCNTs. The structural evolution of the carbon layer and the nucleation and growth process of SWCNTs were monitored in real time with HRTEM images recorded by a CCD camera. 
First principles calculations. Our first-principles calculations were performed within the framework of density functional theory as implemented in the Vienna $\mathrm{Ab}$ initio simulation package $\operatorname{code}^{39,40}$, using the generalized gradient approximation of Perdew-Burke-Ernzerhof ${ }^{41}$. The electron-ion interactions were described using the frozen-core projector augmented wave approach ${ }^{42,43}$. We used a $420 \mathrm{eV}$ cut-off energy for the plane-wave basis set expansion. The k-space sampling was restricted to the $\Gamma$-point. The climbing image nudged elastic band ${ }^{44-46}$ method was used to study the minimum energy path and the corresponding migration barriers for carbon atoms diffusion on an open/closed $\mathrm{BN}$ edge. A damped molecular dynamics was used to relax ions until the force in each image is less than $0.03 \mathrm{eV} / \mathrm{A}$.

1. Baughman, R. H., Zakhidov, A. A. \& de Heer, W. A. Carbon Nanotubes-the Route Toward Applications. Science 297, 787-792 (2002).

2. Hersam, M. C. Progress towards monodisperse single-walled carbon nanotubes. Nat. Nanotechnol. 3, 387-394 (2008).

3. Ding, F., Bolton, K. \& Rosen, A. Nucleation and Growth of Single-Walled Carbon Nanotubes: A Molecular Dynamics Study. J. Phys. Chem. B 108, 17369-17377 (2004).

4. Ding, F., Rosen, A. \& Bolton, K. Molecular dynamics study of the catalyst particle size dependence on carbon nanotube growth. J. Chem. Phys. 121, 2775-2779 (2004)

5. Ding, F. et al. The Importance of Strong Carbon-Metal Adhesion for Catalytic Nucleation of Single-Walled Carbon Nanotubes. Nano Lett. 8, 463-468 (2007).

6. Kong, J., Soh, H. T., Cassell, A. M., Quate, C. F. \& Dai, H. Synthesis of individual single-walled carbon nanotubes on patterned silicon wafers. Nature 395, 878-881 (1998).

7. Hata, K. et al. Water-Assisted Highly Efficient Synthesis of Impurity-Free SingleWalled Carbon Nanotubes. Science 306, 1362-1364 (2004).

8. Huang, S., Cai, X. \& Liu, J. Growth of Millimeter-Long and Horizontally Aligned Single-Walled Carbon Nanotubes on Flat Substrates. J. Am. Chem. Soc. 125, 5636-5637 (2003)

9. Li, Y. et al. Growth of Single-Walled Carbon Nanotubes from Discrete Catalytic Nanoparticles of Various Sizes. J. Phys. Chem. B 105, 11424-11431 (2001).

10. Kim, W. et al. Synthesis of Ultralong and High Percentage of Semiconducting Single-walled Carbon Nanotubes. Nano Lett. 2, 703-708 (2002).

11. Li, Y. et al. Preferential Growth of Semiconducting Single-Walled Carbon Nanotubes by a Plasma Enhanced CVD Method. Nano Lett. 4, 317-321 (2004).

12. Qu, L., Du, F. \& Dai, L. Preferential Syntheses of Semiconducting Vertically Aligned Single-Walled Carbon Nanotubes for Direct Use in FETs. Nano Lett. 8, 2682-2687 (2008).

13. Harutyunyan, A. R. et al. Preferential Growth of Single-Walled Carbon Nanotubes with Metallic Conductivity. Science 326, 116-120 (2009).

14. Zoican Loebick, C. et al. Selective Synthesis of Subnanometer Diameter Semiconducting Single-Walled Carbon Nanotubes. J. Am. Chem. Soc. 132, 11125-11131 (2010).

15. Bachilo, S. M. et al. Narrow (n,m)-Distribution of Single-Walled Carbon Nanotubes Grown Using a Solid Supported Catalyst. J. Am. Chem. Soc. 125, 11186-11187 (2003).

16. Li, X. et al. Selective Synthesis Combined with Chemical Separation of SingleWalled Carbon Nanotubes for Chirality Selection. J. Am. Chem. Soc. 129, 15770-15771 (2007).

17. Ghorannevis, Z., Kato, T., Kaneko, T. \& Hatakeyama, R. Narrow-Chirality Distributed Single-Walled Carbon Nanotube Growth from Nonmagnetic Catalyst. J. Am. Chem. Soc. 132, 9570-9572 (2010).

18. He, M. et al. Predominant $(6,5)$ Single-Walled Carbon Nanotube Growth on a Copper-Promoted Iron Catalyst. J. Am. Chem. Soc. 132, 13994-13996 (2010).

19. Takagi, D., Kobayashi, Y. \& Homma, Y. Carbon Nanotube Growth from Diamond. J. Am. Chem. Soc. 131, 6922-6923 (2009).

20. Yu, X. et al. Cap Formation Engineering: From Opened C60 to Single-Walled Carbon Nanotubes. Nano Lett. 10, 3343-3349 (2010).

21. Yao, Y., Feng, C., Zhang, J. \& Liu, Z. “Cloning” of Single-Walled Carbon Nanotubes via Open-End Growth Mechanism. Nano Lett. 9, 1673-1677 (2009).

22. Tang, D. M., Liu, C. \& Cheng, H. M. Platelet boron nitride nanowires. NANO 1, 65-71 (2006).

23. Tang, D. M., Liu, C. \& Cheng, H. M. Controlled synthesis of quasi-onedimensional boron nitride nanostructures. J. Mater. Res. 22, 2809-2816 (2007).

24. Tang, D. M. et al. Structural evolution of carbon microcoils induced by a direct current. Carbon 47, 670-674 (2009).

25. Tang, D.-M. et al. Carbon nanotube-clamped metal atomic chain. Proc. Natl. Acad. Sci. USA 107, 9055-9059 (2010).

26. Liu, B. et al. Importance of Oxygen in the Metal-Free Catalytic Growth of SingleWalled Carbon Nanotubes from SiOx by a Vapor-Solid-Solid Mechanism. J. Am. Chem. Soc. 133, 197-199 (2011).

27. Bachilo, S. M. et al. Structure-assigned optical spectra of single-walled carbon nanotubes. Science 298, 2361-2366 (2002).
28. Gómez-Gualdrón, D. A. \& Balbuena, P. B. Effect of Metal Cluster-Cap Interactions on the Catalyzed Growth of Single-Wall Carbon Nanotubes. J. Phys. Chem. C 113, 698-709 (2008).

29. Chiang, W.-H. \& Mohan Sankaran, R. Linking catalyst composition to chirality distributions of as-grown single-walled carbon nanotubes by tuning NixFe1-x nanoparticles. Nat. Mater. 8, 882-886 (2009).

30. Reich, S., Li, L. \& Robertson, J. Control the chirality of carbon nanotubes by epitaxial growth. Chem. Phys. Lett. 421, 469-472 (2006).

31. Helveg, S. et al. Atomic-scale imaging of carbon nanofibre growth. Nature 427, 426-429 (2004)

32. Sharma, R. \& Iqbal, Z. In situ observations of carbon nanotube formation using environmental transmission electron microscopy. Appl. Phys. Lett. 84, 990-992 (2004).

33. Lin, M. et al. Direct observation of single-walled carbon nanotube growth at the atomistic scale. Nano Lett. 6, 449-452 (2006).

34. Lin, M. et al. Dynamical observation of bamboo-like carbon nanotube growth. Nano Lett. 7, 2234-2238 (2007).

35. Yoshida, H., Takeda, S., Uchiyama, T., Kohno, H. \& Homma, Y. Atomic-scale insitu observation of carbon nanotube growth from solid state iron carbide nanoparticles. Nano Lett. 8, 2082-2086 (2008).

36. Novoselov, K. S. et al. Two-dimensional atomic crystals. Proc. Natl. Acad. Sci. USA 102, 10451-10453 (2005).

37. Coleman, J. N. et al. Two-Dimensional Nanosheets Produced by Liquid Exfoliation of Layered Materials. Science 331, 568-571 (2011).

38. Osada, M. \& Sasaki, T. Two-Dimensional Dielectric Nanosheets: Novel Nanoelectronics From Nanocrystal Building Blocks. Adv. Mater. 24, 210-228 (2012).

39. Kresse, G. \& Furthmüller, J. Efficiency of ab-initio total energy calculations for metals and semiconductors using a plane-wave basis set. Comput. Mater. Sci. 6, 15-50 (1996).

40. Kresse, G. \& Furthmüllerr, J. Efficient iterative schemes for ab initio total-energy calculations using a plane-wave basis set. Phys. Rev. B 54, 11169 (1996).

41. Perdew, J. P., Burke, K. \& Ernzerhof, M. Generalized Gradient Approximation Made Simple. Phys. Rev. Lett. 77, 3865 (1996).

42. Blöchl, P. E. Projector augmented-wave method. Phys. Rev. B 50, 17953 (1994).

43. Kresse, G. \& Joubert, D. From ultrasoft pseudopotentials to the projector augmented-wave method. Phys. Rev. B 59, 1758 (1999).

44. Henkelman, G., Uberuaga, B. P. \& Jónsson, H. A climbing image nudged elastic band method for finding saddle points and minimum energy paths. J. Chem. Phys. 113, 9901-9904 (2000).

45. Henkelman, G. \& Jónsson, H. Improved tangent estimate in the nudged elastic band method for finding minimum energy paths and saddle points. J. Chem. Phys. 113, 9978-9985 (2000)

46. Sheppard, D., Terrell, R. \& Henkelman, G. Optimization methods for finding minimum energy paths. J. Chem. Phys. 128, 134106-134110 (2008).

\section{Acknowledgments}

TEM support from Dr. Chuan-Bin Jiang and Mr. Bo Wu is acknowledged. The authors thank Dr. Li-Bo Gao for helpful discussion and suggestions. This work is supported by the MOST of China (2011CB932601) and NSFC (50921004, 51102242, and 51272257). L.C.Y. acknowledges the grants from Informalization Construction Project of CAS (No. INFO-115-B01) and the GPU Project of MOF (No. ZDYZ2008-2-A12), China.

\section{Author contributions}

D.M.T., L.L.Z., C.L. and H.M.C. conceived and designed the experiments. D.M.T., L.L.Z., H.J., Z.Z. and C.L. carried out the experiments. L.C.Y. conducted the first principles calculations. D.M.T., L.L.Z., L.C.Y., C.L. and H.M.C. wrote the paper. E.I.K., C.L. and H.M.C. supervised the project. All authors contributed to data analysis and scientific discussions.

\section{Additional information}

Supplementary information accompanies this paper at http://www.nature.com/ scientificreports

Competing financial interests: The authors declare no competing financial interests.

License: This work is licensed under a Creative Commons

Attribution-NonCommercial-NoDerivs 3.0 Unported License. To view a copy of this license, visit http://creativecommons.org/licenses/by-nc-nd/3.0/

How to cite this article: Tang, D.M. et al. Heteroepitaxial Growth of Single-Walled Carbon Nanotubes from Boron Nitride. Sci. Rep. 2, 971; DOI:10.1038/srep00971 (2012). 